\title{
Electric signal detection system based on dynamic programming location residual error repair control \\ CHEN Yubo
}

Beijing university of posts and Telecommunications, China, 100876

Keywords: dynamic programming; residual error repair; control; signal; detecting system

\begin{abstract}
Accurate measurement of flow has been used in energy saving, consumption reduction, economic calculation, automatic control and other aspects. In micro flow measurement, measurement method of floater flow plays a very important role. A kind of flow measurement system which is applicable to detection of micro flow signal has been proposed in this paper, which is based on measurement principle of floater flow to convert the change of floater displacement into voltage change through mutual inductance with application of differential transformer displacement transducer. Signal conditioning circuit has been designed to process weak voltage signal from the transducer so that requirements of single chip microcomputer (SCM) to input signal can be met after enlarging and filtering it. Intelligent SCM software and hardware system has been designed with function extension of the system so that the complete system can not only accurately reflect the measured flow size in real time but also give alarm when flow range is beyond the normal value. Finally, communication module circuit that can realize transmission of flow information between SCM and computer has been designed, which has provided conditions for the intelligent remote control of system.
\end{abstract}

\section{Introduction}

Flow is the quantity of fluid passing a certain area of section in the unit time. If the quantity is expressed with volume of fluid, it will be called as instantaneous volume flow and called volume flow for short; if it is expressed with quality of flow, it will be called as instantaneous quality flow and called quality flow for short. During this period, the cumulative value of volume flow or quality flow of fluid is called as accumulative flow. Flow measurement of the flowing fluid in a certain channel is collectively known as flow metering. Fluid in flow measurement is diverse, for example, measured objects include air, liquid, mixed fluid; fluid temperature, pressure, flow are different greatly and measurement accuracies required are different, too. Thus, flow measurement task is to research various corresponding measurement methods based on measurement purpose, type and flowing state of measured fluid, measurement site and other measuring conditions, ensuring the correct transmission of the flow measurement value.

\section{Dynamic programming location residual error repair control}

If flowing of the fluid does not change with time and it is steady flow, the flow can be expressed with volume or quality of fluid passing a certain section in unit time. When flowing of the fluid changes with time and it is not steady flow, the flow continues to change with time at this time. Thus, it can be assumed that the flowing is constant during a micro $\Delta t$ time around this moment, and the flow at a moment can be expressed with volume or quality of fluid passing in the micro time interval.

Assumed that a micro area in section passed by fluid is $d F$ and select the flow velocity of fluid passing the micro area as $u$, then volume flow $d q$ of fluid passing the micro area $d F$ is:

$$
d q=u d F
$$

Volume flow q of fluid passing the complete selection area can be acquired with integral of sectional area F: 


$$
q=\int u d F
$$

Quality flow can be expressed with product of fluid volume flow and fluid density. If quality flow is $Q$ and fluid density is $\rho$, then:

$$
Q=\rho q=\rho u F
$$

In above formula, unit of volume flow is $\mathrm{m} 3 / \mathrm{s}^{\mathrm{or}} \mathrm{m} 3 / \mathrm{h}$; unit of quality flow is $\mathrm{kg} / \mathrm{s}$ or $\mathrm{kg} / \mathrm{h}$.

In engineering application, total fluid quantity passing the pipe during a period is often required to be measured at the same time, namely the accumulative flow of fluid passing pipe is required to be measured. If fluid volume flow is $Q_{V}$ and quality volume is $Q_{m}$, then accumulative flow of fluid passing in the time interval $\Delta \mathrm{t}$ can be expressed with the following formula:

Accumulative flow of fluid quality:

$$
Q_{m}=\int q_{m} d t
$$

Accumulative flow of fluid volume:

$$
Q_{v}=\int q_{v} d t
$$

Unit of accumulative volume flow is $\mathrm{m} 3$ and that of accumulative quality flow is $\mathrm{kg}$.

Thus, if flowing is the steady flow and fluid density is $\rho$, then it can be obtained from above formula:

$$
Q_{m}=q_{m} t=\rho q_{v} t=\rho Q_{v}
$$

It can be seen from above that measurement of accumulative flow is the measurement of fluid volume or quality.

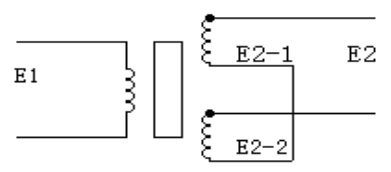

Fig. 1 Equivalent Circuit Diagram of Differential Transformer

From the figure, it can be seen that differential transformer consists of armature, primary coil, secondary coil and coil frame. Primary coil is used as an excitation of differential transformer, which is equivalent to the primary side of the transformer; while secondary coil consists of two coils with same structure size and parameter which are in tandem connection with reverse polarity, and it is equivalent to the secondary side of the transformer. Thus, it can be thought that structure of differential transformer can be equivalent to general transformer. But the structure difference is: general transformer is fitted with closed magnetic circuit while differential transformer is fitted with opened magnetic circuit; mutual inductance between primary and secondary side of general transformer is constant (magnetic circuit size specified) while mutual inductance between primary and secondary side of differential transformer has corresponding change with movement of armature. Relative position of armature is reflected with change of mutual inductance for differential transformer.

Residual voltage at zero point will cause insensitive zone near the zero point and error in measurement. Traditional method to remove residual voltage at zero point is usually to add phase-sensitive rectification circuit in front of amplifying circuit or adopt compensating circuit. However, the practical value is little in practical application and it makes circuit structure and later commissioning complex. In flow measurement system, we directly input output voltage of the secondary coil of differential transformer into differential amplifier and realize the effect of removing residual voltage at zero point by using inhibiting effect of differential amplifier on common-mode signal. At the same time, effect of signal phase adjustment and amplification has been realized so that circuit can be simplified.

Design of active low-pass filter is to determine the order $\mathrm{n}$ of filter and select specific circuit form to work out specific numerical values of various components in the circuit for installation and 
commissioning of circuit based on the given index requirements so that designed filter can meet index requirements, and the specific procedures are as follows:

(1)Determine the order $\mathrm{n}$ of filter based on requirement of stopband attenuation rate.

(2)Select specific circuit form.

(3)Establish coefficient equation set according to denominator polynomials of transfer functions of circuit and normalized filter.

(4)Solve the equation set to acquire specific numerical values of components in the circuit.

(5)Install the circuit and implement commissioning so that circuit performance can meets index requirements.

The maximum frequency-response curve of Butterworth filter in passband is flat without fluctuation while it gradually decreases to zero in suppressed frequency band, so Butterworth filter is selected according to requirement of system design. Since attenuation from passband to stopband for first-order Butterworth filter is slow and design of high-order Butterworth filter is complex with many components required, second-order Butterworth filter circuit is selected here.

Principle of active second-order low-pass filter is shown as Fig. 2 and it is a kind of common second-order filter circuit of voltage controlled voltage source.

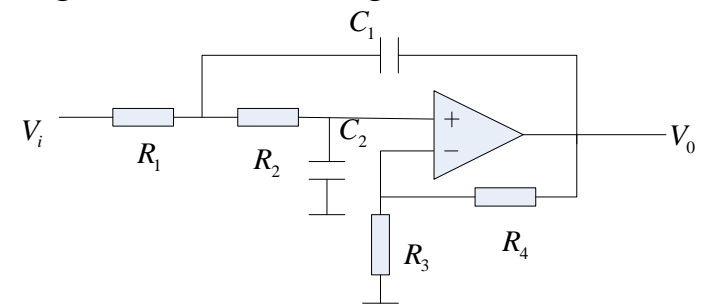

Fig. 2 Schematic Diagram of Active Low-pass Filter

Normalized transfer function of the second-order Butterworth low-pass filter can be expressed as the ratio of two polynomials:

$$
A(s)=\frac{A_{V} \omega_{c}^{2}}{s^{2}+\frac{\omega_{c}}{Q} s+\omega_{c}^{2}}
$$

Where Av is voltage amplification factor in passband and $\omega \mathrm{C}$ is cut-off angular frequency, with $\mathrm{Q}$ as quality factor, and among them,

$$
\begin{aligned}
& \omega_{c}=\frac{1}{\sqrt{R_{1} R_{2} C_{1} C_{2}}}=2 \pi f_{c} \\
& \qquad A_{V}=1+\frac{R_{4}}{R_{3}}
\end{aligned}
$$

\section{Detecting system design}

$12 \mathrm{~V}$ DC stabilized-voltage switching power supply can be used as power supply of transducer for direct supply. However, $12 \mathrm{~V}$ voltages can not be supplied for SCM system at the same time, thus, LM2940 is used in SCM system for power supply. LM2940 is low drop-out three terminal regulator with fixed output voltage; output voltage is $5 \mathrm{~V}$ and output current is $1 \mathrm{~A}$; when output current is $1 \mathrm{~A}$, the minimum input and output voltage difference is less than $0.8 \mathrm{~V}$; the maximum input voltage is $26 \mathrm{~V}$; working temperature is from -40 to $+125^{\circ} \mathrm{C}$; it includes quiescent current reduction circuit, current limiting, thermal protection, reverse battery and reverse insert protection circuit. LM2940 is used as voltage stabilizing chip in this module, with $12 \mathrm{~V}$ input voltage and $+5 \mathrm{~V}$ output voltage, which provides $+5 \mathrm{~V}$ stable power supply for the complete system. The design can improve stability of the system and reduce working environment requirements of the system. Button with lock is added into circuit so that power supply can be cut when stopping work to save electric energy and LED indicator lamps are added at input and output ends to indicate working state of power supply. The circuit diagram is shown as Fig. 3-5. 


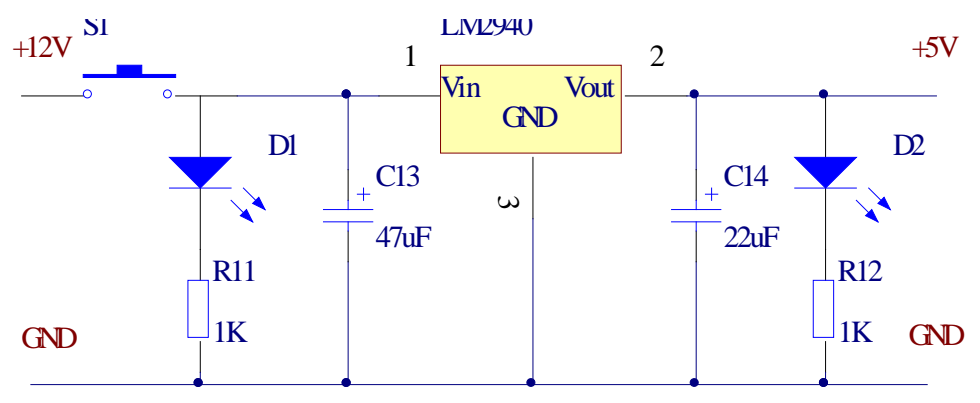

Fig. 3 Power Module

\section{Conclusion}

In this paper, differential transformer circuit, signal conditioning circuit, SCM software and hardware system design and other problems based on floater displacement measurement in micro flow signal detecting system have been discussed completely. Aiming at micro flow signal detecting problem, the main works in this paper are as follows: 1. Use armature embedded in floater to convert the change of floater displacement into change of output voltage of differential transformer displacement transducer through mutual inductance so that flow of liquid measured can be reflected correctly. 2. AT89S52 has been selected as micro controller and software and hardware of the system have been designed. Peripheral circuit is composed of signal conditioning circuit, power module circuit, LCD display circuit, communication circuit and buzzer alarm circuit, with more perfect functions. 3 Transmission and round trip of system signal has been decreased and working reliability of the system has been improved since system structure has been simplified and equipment connection has been reduced.

\section{Reference}

[1] Svärd C, Nyberg M, Frisk E, et al. Data-driven and adaptive statistical residual evaluation for fault detection with an automotive application[J]. Mechanical Systems \& Signal Processing, 2012, 45(1):170-192.

[2] Mangoubi R, Desai M, Edelmayer A, et al. Robust Detection and Estimation in Dynamic Systems and Statistical Signal Processing: Intersections, Parallel Paths and Applications[J]. European Journal of Control, 2009, 15(3-4):348-369.

[3] Redding J, Dydek Z, How J P, et al. Proactive planning for persistent missions using composite model-reference adaptive control and approximate dynamic programming[J]. 2011:2332-2337.

[4] Ney H. The use of a one-stage dynamic programming algorithm for connected word recognition[J]. Acoustics Speech \& Signal Processing IEEE Transactions on, 1984, 32(2):263-271.

[5] Wang Y, Shi P, Li K, et al. An energy efficient medium access control protocol for target tracking based on dynamic convey tree collaboration in wireless sensor networks[J]. International Journal of Communication Systems, 2012, 25(9):1139-1159. 